\title{
6. AN IMPROVED IMPREGNATION TECHNIQUE FOR STUDYING STRUCTURE OF UNLITHIFIED COHESIVE SEDIMENTS ${ }^{1}$
}

\author{
James F. Swartz ${ }^{2}$ and Nancy Lindsley-Griffin ${ }^{3}$
}

\begin{abstract}
Impermeable, fragile sediment samples can be successfully impregnated for petrographic study by treating them with a low-viscosity epoxy resin. Before impregnation in the epoxy resin, samples are soaked in acetone until water has been completely removed. Samples are soaked in resin for several days, then cured in an oven at $50^{\circ}$ and $70^{\circ} \mathrm{C}$. The whole procedure takes 6 to 10 days. After hardening, samples are prepared for study using conventional techniques. Microstructures such as mud veins, microfaults, and delicate primary textures can be preserved well using this technique.
\end{abstract}

\section{INTRODUCTION}

Samples of soft, wet sediments were collected during Ocean Drilling Program (ODP) Leg 112 to the Peru margin for shore-based microstructural studies. An impregnation technique had to be developed to preserve the original textures and structures and yet allow us to prepare petrographic thin sections. Our major goal was to study the mud veins and mud-injected microfaults (PI. 1) that were recovered in abundance during Leg 112 (Lindsley-Griffin et al., this volume).

We developed this process by adapting techniques described by Spurr (1969), Conway (1982), and Jim (1985) to the special requirements of the Leg 112 samples. Most of our samples were very wet, relatively impermeable diatomaceous oozes and muds, whereas previously developed techniques were for moist to dry clay-rich soil samples. The use of acetone to replace moisture was suggested by Conway (1982) and by Jim (1985), and the low-viscosity resin was developed originally by Spurr (1969). Our modified impregnation process works well for wet to dry sediments, and can also be applied to rock chips and semilithified sediments of irregular shape. The whole procedure takes 6 to 10 days and requires a minimum of equipment.

Most of our samples consisted of oriented cubes of finegrained diatomaceous muds in plastic boxes measuring $2 \mathrm{~cm}$ on the side, of the type used for shipboard paleomagnetic samples. Direction of the core top was indicated on these boxes by an arrow drawn in indelible ink. Samples were collected shortly after core recovery by placing the open box over the desired sample area and hammering it into the core until the box was filled with sediment. The box was then lifted out of the surrounding sediment with a spatula, capped, and sealed in a plastic bag for shipment. One must seal the plastic bags properly when the samples are originally collected, because uncontrolled desiccation of samples in improperly sealed bags may partially destroy the original textures and structures.

\section{Resin Used for Impregnation}

As noted by Jim (1985), standard resins are too viscous to infiltrate impermeable fine-grained sediments. We needed a

\footnotetext{
${ }^{1}$ Suess, E., von Huene, R., et al., 1990. Proc. ODP, Sci. Results, 112: College Station, TX (Ocean Drilling Program).

2 Department of Geology, Univ. of Nebraska, Lincoln, NE 68588; now at School of Geology and Geophysics, Univ, of Oklahoma, Norman, OK 73019.

${ }^{3}$ Department of Geology, Univ. of Nebraska, Lincoln, NE 68588.
}

resin having very low viscosity to penetrate the sample cubes completely (Pl. 1, Figs. 1, 2). Such a resin was developed by Spurr (1969) to embed biological materials for electron microscopy. Spurr resin components are available separately or as kits from Ernest F. Fullam, Inc., 900 Albany Shaker Road, Latham, NY 12110.

Different proportions of each of the four components in this epoxy resin allow for variations in viscosity, pot life, and final hardness. For the Leg 112 samples, we used proportions that combine extra long pot life with low viscosity (Table 1); other combinations and their properties have been tabulated by Spurr (1969). The technique of mixing the components and the precautions that should be taken in handling the resin were described in detail by Jim (1985). Spurr (1969) recommended using medicine dropper pipettes for dispensing the components, although we found that syringes are just as effective and are easier to clean. Prepared resin should be stored in a freezer to deter partial polymerization and to extend the pot life for up to several months. Storage containers should be sealed tightly to prevent the hygroscopic resin from absorbing moisture from humid air.

Table 1. Proportions of Spurr resin components for long pot life and low viscosity.

\begin{tabular}{lc}
\hline \multicolumn{1}{c}{ Component } & Proportion (g) \\
\hline ERL-4206 (Vinylcyclohexane dioxide) & 10.0 \\
D.E.R. 736 (Diglycidyl ether of & 6.0 \\
polypropyleneglycol) & 26.0 \\
NSA (Nonenyl succinic anhydride) & 0.2 \\
DMAE (Dimethylaminoethanol) & \\
\hline
\end{tabular}

\section{PREPARATION OF SAMPLES FOR IMPREGNATION}

Wet or moist samples must be soaked in acetone to replace the water before resin impregnation, as suggested by Conway (1982) and Jim (1985). For dry samples, impregnation works best if they are first soaked in acetone to "wet" the grain boundaries. We found that placing very wet samples in acetone caused sample disruption and expulsion of sediment during acetone treatment. We allowed such samples to air-dry slightly by perforating the plastic bag in which they were packed. In cases where minor desiccation cracks formed during drying, the original fabrics and structures were preserved between cracks (Pl. 1, Figs. 3, 4). As pointed out by 
Conway (1982), acetone treatment before impregnation effectively reduces shrinking and cracking. Samples that contain significant amounts of clay might undergo excessive shrinkage during this partial drying step, but the Leg 112 samples are all diatomaceous oozes and muds in which clay minerals are a minor component. We did not observe excessive shrinkage or distortion in any of the Leg 112 samples.

Attempts to use vacuum impregnation without acetone treatment were unsuccessful because the resin could not penetrate to the center of the sample cube (Pl. 2, Fig. 1). Vacuum impregnation of both wet and dry samples (using commercially available equipment) caused the samples to disaggregate, rendering them useless. The behavior of these samples during vacuum impregnation is evidence of the low permeability of these diatomaceous muds and oozes, which may be a factor in the development of mud-filled dewatering veins (Lindsley-Griffin et al., this volume).

The semidry samples are prepared for soaking in acetone by removing the cap of the plastic sample box and inserting a small steel pin through the sediment "top" to preserve the sample orientation during all stages of processing. The head of this pin is just visible in Plate 2, Figure 2, at the left of the sample cube. When the impregnated sample cubes are sawed into slabs, the pin is preserved as a tiny round speck at one side of the slab-too small to be seen in the slabs in Plate 2, Figure 3 , but easily visible in bright light because of its high reflectivity.

Next, the plastic sample boxes are wrapped in heavy aluminum foil, leaving only the uncapped open surface of the sediment exposed. Both of these initial preparation steps are necessary because the plastic sample box loses its strength when placed in acetone, taking on a consistency similar to rubber cement, and the original orientation marks on the box are lost upon contact with acetone.

\section{Acetone Treatment}

After the samples are marked and wrapped, they are placed in individual $50-\mathrm{cm}^{3}$ lidded glass jars marked with the sample number. These jars are filled with acetone, capped, and the samples are soaked for three to five days. This step removes moisture from the samples and enhances the ability of the resin to migrate into the sample. If the samples are very wet to begin with, one must change the acetone several times during the soaking process, until water no longer accumulates in the beaker (Conway, 1982; Jim, 1985). Dry copper sulfate, added to the acetone residue, will turn dark blue if even a small quantity of water remains. All moisture must be removed from the sediment before attempting resin transfer because of the resin's hygroscopic character.

One drawback of our method is that the plastic sample boxes used by ODP dissolve during acetone treatment, and a film of soft plastic collects on top of the samples. During and after soaking, this should be scraped off to maximize the surface area available for acetone infiltration and resin impregnation. The plastic can be easily removed with a spatula after the sample has been exposed to the air for 30 to $60 \mathrm{~s}$. Samples that are firm enough to hold their shape can be placed on their sides in the acetone to allow most of the soft plastic to flow out under the influence of gravity.

Once the water has been completely expelled, the samples can be removed from the acetone and prepared for impregnation. Each sample is wrapped in a sheet of polyethylene (commercially available food wrap) and a second layer of aluminum foil. This minimizes the amount of resin lost by leakage. A small lip should be left around the top of the sample to act as a resin reservoir (PI. 2, Fig. 2), thereby decreasing the number of times resin must be added to the sample. Acetone treatment can eliminated if the sample consists of dry, permeable sand or coarse silt. The sample shown in Plate 2, Figure 3 , was successfully impregnated without acetone treatment; the original plastic box can still be seen around the sediment. However, the center of this sample did not become impregnated as completely as the darker rim of the sample, and the thin section made from this sample was extensively plucked during grinding. We conclude that better results might have been obtained if this sample had been soaked in acetone to "wet" the grains before impregnation.

\section{IMPREGNATION PROCESS}

To begin impregnation, the acetone-soaked samples are covered with resin to the top of the foil lip (Pl. 1, Fig. 2) and placed in dry, lidded jars. The resin directly replaces the acetone in the sample over a period of several days. Samples can be left in the resin for up to a week because it polymerizes slowly. During the first few days of impregnation, resin must be added regularly, and the residue of acetone and resin that collects in the bottom of the jars must be poured off.

Most of the transfer is complete in four days, after which the samples are placed under vacuum to enhance further resin absorption. A simple technique for this is to place the uncapped sample jars in a bell jar and draw a vacuum for approximately $1 \mathrm{hr}$. The bell jar is then sealed, and the samples are left under vacuum for one day. The samples are removed from the bell jar and allowed to soak in the resin for several more hours before being placed in an oven to cure the resin. Other scientists have suggested performing the entire impregnation under vacuum (Jim, 1985), but we obtained better results and less sample disruption by partially impregnating the samples before subjecting them to vacuum.

\section{CURING AND CUTTING SAMPLES}

Once impregnation is complete, the samples are placed in an oven and cured in two stages to prevent cracking caused by rapid polymerization. We heated the samples for $24 \mathrm{hr}$ at $50^{\circ} \mathrm{C}$, then at $70^{\circ} \mathrm{C}$ for another $24 \mathrm{hr}$, as suggested by the manufacturer of the resin. If cracking occurs, the curing process can be extended; Jim (1985) recommends four stages of curing, from $30^{\circ}$ to $60^{\circ} \mathrm{C}$.

After curing, the samples are removed and allowed to cool to room temperature. Then, the samples are cut into thin slices using a side arm thin-section saw. As the aluminum foil inhibits slicing the samples, it should be first removed as completely as possible. The impregnated sample cubes can be sliced into three or four sections, each $3 \mathrm{~mm}$ thick (Pl. 2, Figs. $3,4)$. These slabs can be made into standard petrographic thin sections, or they may be polished for study with an energy dispersive system (EDS) or reflecting microscope.

\section{Preparation of Thin Sections}

The use of commercially available automatic thin-section equipment produced excessive plucking of individual grains, or else plucked the entire slab off the glass. The variation of Spurr resin that we used produces a relatively soft impregnated sample; final hardness was decreased to obtain minimum viscosity and maximum penetration of the resin. Sediment samples that are more permeable than the Leg 112 samples might be impregnated well with one of the lower viscosity mixtures that produces a firmer end product (Spurr, 1969), allowing for automatic thin-section equipment to be used.

We obtained the best results by preparing each thin section individually by hand. Individual preparation of each thin section allowed the section to be monitored closely, and grinding was halted if plucking became excessive. For the 
Peru margin sediments, obtaining a precise thickness was less important than in standard petrographic studies because most of these diatomaceous oozes and muds are optically isotropic.

We found that preparation of thin sections from our slabs of impregnated samples requires special care when gluing the sample to the slide. Instead of using the normal, thin, uniform coating of glue, a large quantity of glue is placed on both slide and sample. The sample slab is then gently placed on the slide and swirled around to push out air bubbles. Once the sample has been positioned on the slide, gentle pressure is applied in the center of the sample with a pencil eraser to distribute the glue uniformly. Too much pressure causes the formation of air pockets that may lead to cracking of the slide upon cooling. If air bubbles are small and few, they can be ignored. After heating, the slides should be cooled slowly on a hot plate to help prevent cracking. After cooling overnight, the sections are ground on a side-arm grinder until they are just thin enough for light to pass through and illuminate the structures. During grinding, the sections must be monitored carefully for any wedging or thinning due to an uneven glue layer. If wedging does occur, the sample should be ground from another angle to achieve a more uniform thickness.

\section{SUMMARY}

Cubes of soft diatomaceous muds collected during ODP Leg 112 for microstructural study proved difficult to impregnate for petrographic thin sections because of their fragile and impermeable nature. Our technique, modified from techniques developed for soil and clay samples (Conrad, 1982; Jim, 1985), uses a low-viscosity epoxy resin (Spurr, 1969) having a long pot life that is available commercially in kit form or as separate components. The samples are soaked in acetone to remove water if they are wet or to moisten the grains if they are dry. Then the samples are soaked in the resin for several days. The resin-impregnated sediment samples are hardened in two stages by heating in an oven at $50^{\circ}$ and $70^{\circ} \mathrm{C}$. The procedure takes six to 10 days to complete. Each $2-\mathrm{cm}$ cube can be sawed into several thin slabs, which can be polished or ground into petrographic thin sections. The main drawback to our technique is that the resin mixture with lowest viscosity does not harden enough for thin sections to be made using automatic thin-section equipment. If one plans to use polished slabs extensively, the Spurr resin can be modified to produce a harder result (Spurr, 1969), but this will change other properties of the resin.

\section{ACKNOWLEDGMENTS}

This work was supported by the U.S. Science Program under the sponsorship of the National Science Foundation and the Joint Oceanographic Institutions Inc. We thank the two anonymous reviewers for their comments, which substantially improved the manuscript.

\section{REFERENCES}

Conway, J. S., 1982. A simplified method for impregnation of soils and similar clay-rich sediments. J. Sediment. Petrol., 52:650-651.

Jim, C. Y., 1985. Impregnation of moist and dry unconsolidated clay samples using Spurr resin for microstructural studies. J. Sediment. Petrol., 55:597-599.

Spurr, A. R., 1969. A low-viscosity epoxy resin embedding medium for electron microscopy. J. Ultrastructure Res., 26:31-43.

Date of initial receipt: 12 October 1988

Date of acceptance: 1 June 1989

Ms 112B-198 

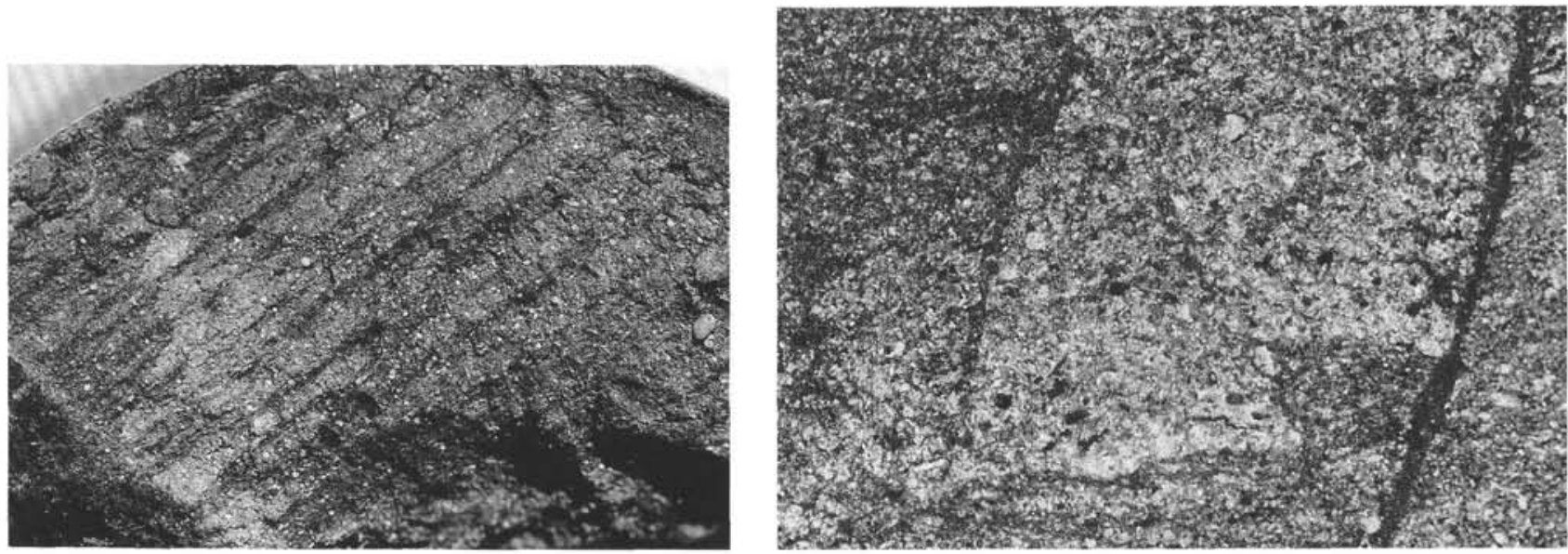

1

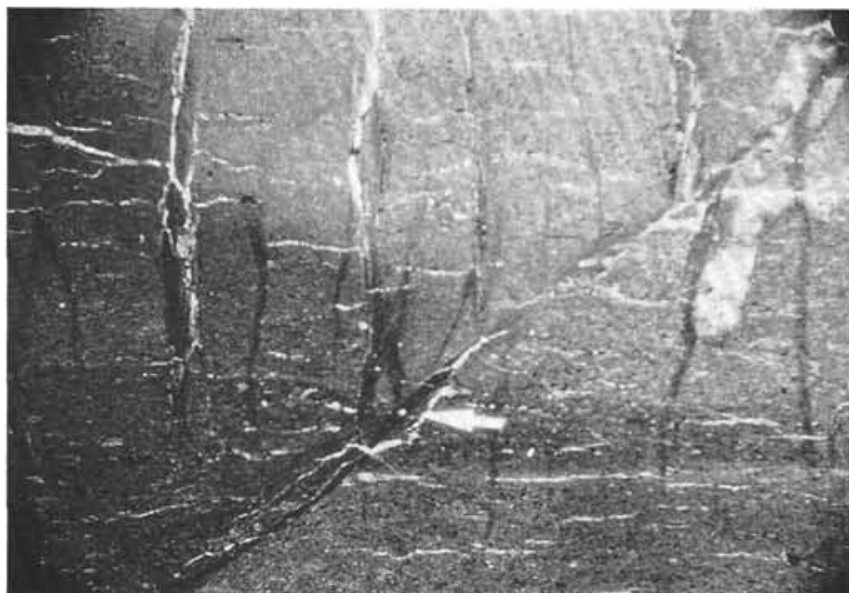

3 $\overline{4 m m}$

2

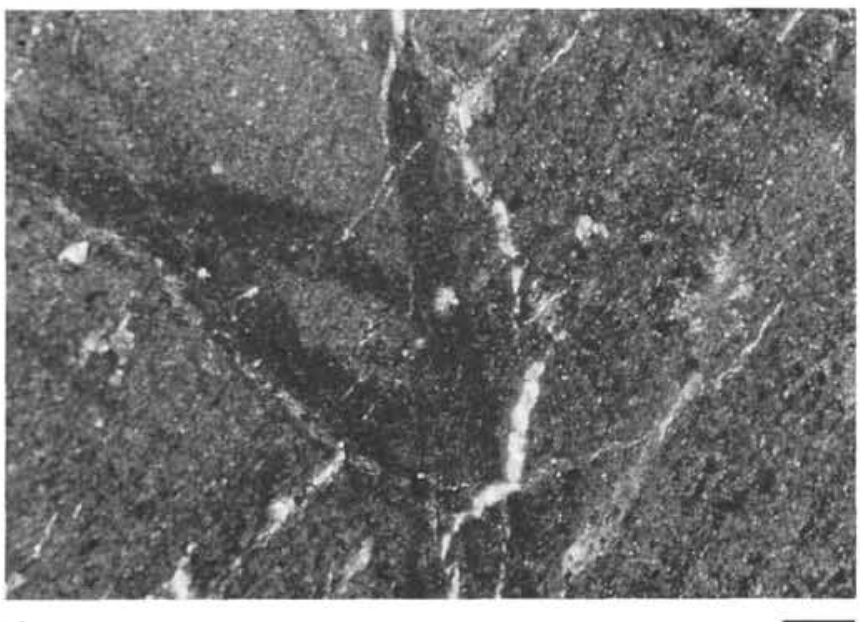

4

$300 \mu \mathrm{m}$

Plate 1. Examples of microstructures. 1. Top of core containing well-developed mud veins in diatomaceous muds and oozes. The low permeability of these sediments and the fragile nature of the microstructures required a special impregnation treatment (Section 112-679A-1H-3). 2. Thin-section photo of two ages of normal faults injected with thin mud veins in diatomaceous mud. When properly performed, this impregnation technique preserves original textures and structural relationships without distortion. Even the delicate internal features of these veins are preserved (Sample 112-688E-32R-1, 80-84 cm; plane light). 3. Thin-section photo of two generations of mud veins, cut by a third-generation mud vein injected along a normal fault. This sample was in an improperly sealed plastic bag and had partially dried out before impregnation, forming desiccation cracks (arrow) (Sample 112-680B-10H-4, 69-71 cm; plane light). 4. Thin-section photo of sample in Figure 3. Closeup view of area near arrow in Figure 3, with a slightly different orientation to enhance preferred orientation of clay minerals within veins. Original structures are well preserved between desiccation cracks, and the crosscutting relationships can be clearly seen. Younger vein is the injection along the microfault, seen as vertical in this view (112-680B-10H-4, 69-71 cm; crossed nicols with gypsum plate). 


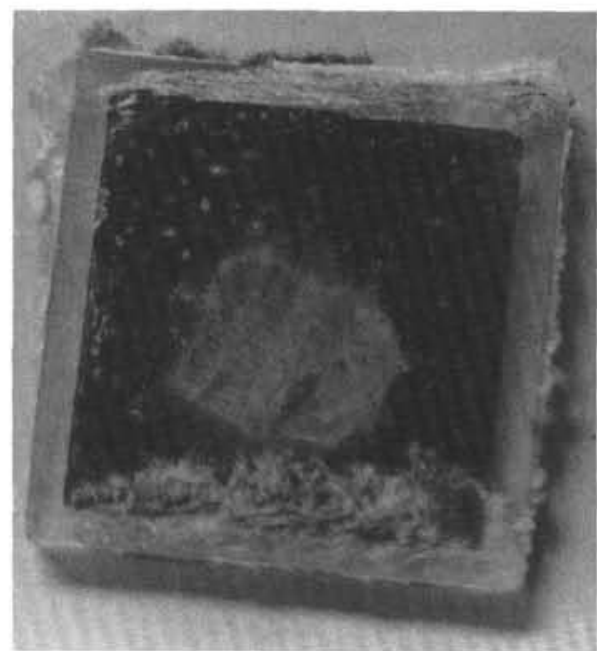

1

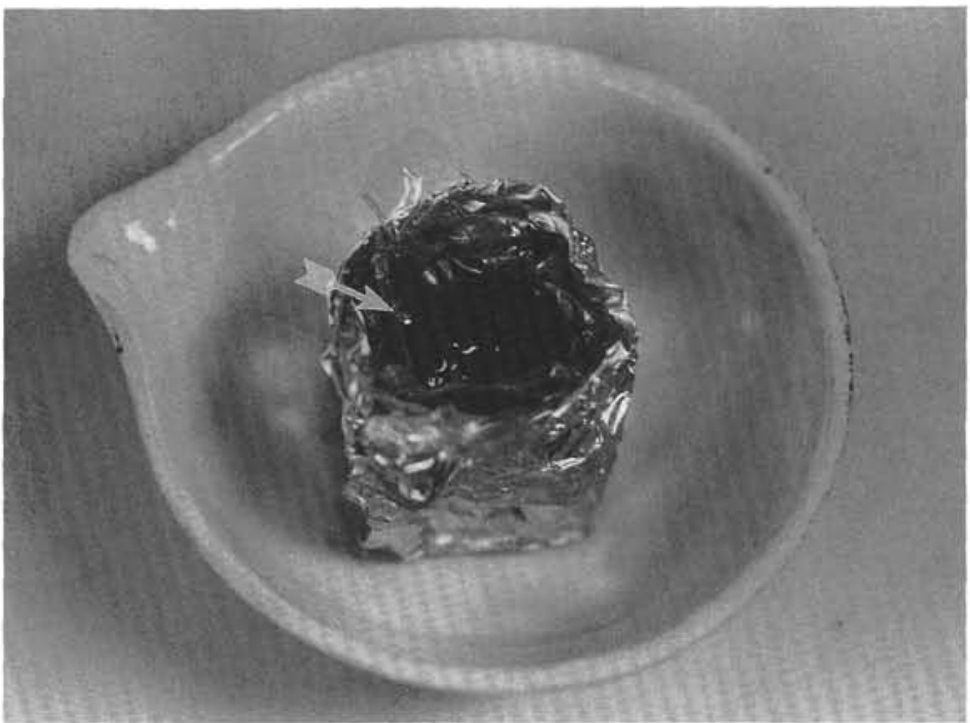

2

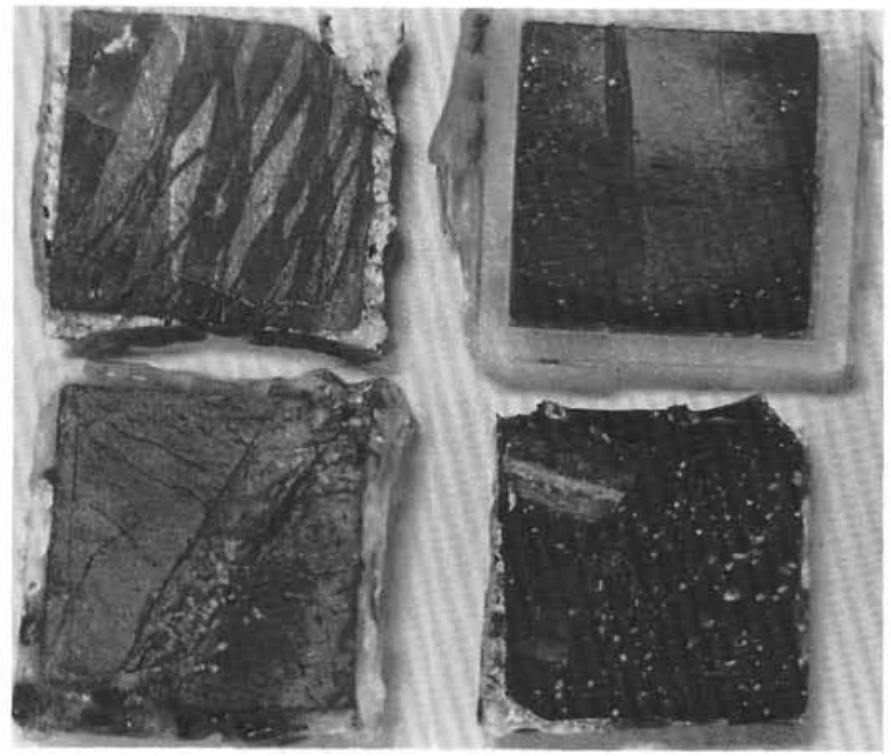

3

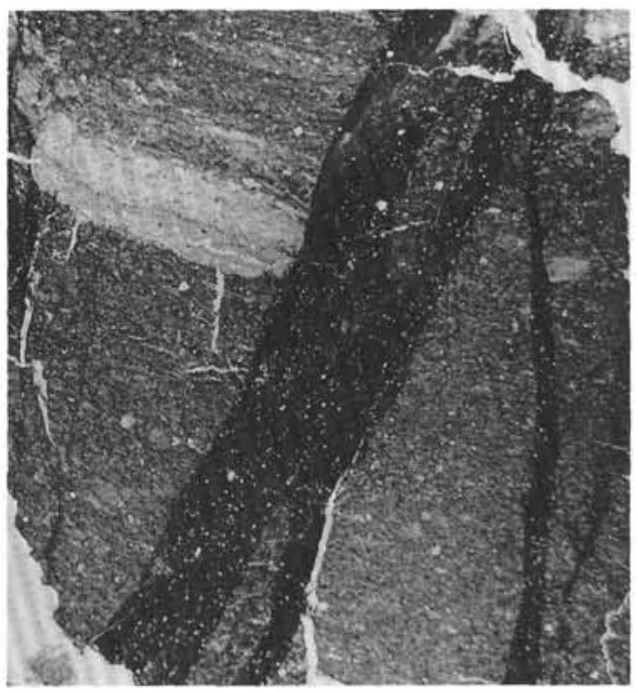

4

$5 \mathrm{~mm}$

Plate 2. 1. Cross-sectional slab of incompletely impregnated sediment cube. Impregnation medium failed to penetrate this relatively impermeable diatomaceous mud, leaving the interior of the sample cube untreated. Slab is $2 \mathrm{~cm}$ wide. 2. Sample cube prepared for resin exchange after acetone treatment. Cube is wrapped in layers of polyethylene and aluminum foil to prevent leakage. A small lip of foil projects above the sample surface to form a reservoir for the resin. Head of steel pin (arrow) indicates sample "top." Sample cube is approximately $2 \mathrm{~cm}$ wide. 3. Sawed slabs of sediment cubes after impregnation. Mud veins can be clearly seen on the surface of the slabs. Slabs are approximately $2 \mathrm{~cm}$ wide (samples, clockwise from upper left: $112-679 \mathrm{D}-10 \mathrm{H}-2,39-41 \mathrm{~cm} ; 112-680 \mathrm{~A}-8 \mathrm{H}-1,83-86 \mathrm{~cm} ; 112-679 \mathrm{D}-5 \mathrm{H}-3,122-124 \mathrm{~cm} ; 112-685 \mathrm{~A}-4 \mathrm{H}-3,74-76 \mathrm{~cm}$ ). 4 . Photomicrograph of an entire thin section made from an impregnated sample. This is the same sample seen in the lower right corner of Figure 3 , but a different slab. Change in shape of mud veins from one slab to the next is typical (Sample 112-679D-5H-3, 122-124 cm). 